\title{
Fractured Metallic Tracheostomy Tube: Report of a Fractographic Study
}

Monthira Tanthanuch, M.D. , Surasak Sangkhathat, M.D., Ph.D. ${ }^{1}$, Wandee Khaimook, M.D. ${ }^{2}$, Sawit Tanthanuch, M.Eng. ${ }^{3}$

1Department of Surgery, Faculty of Medicine, Prince of Songkla University, Hat Yai, Songkhla 90110, Thailand.

${ }^{2}$ Department of Otolaryngology, Faculty of Medicine, Prince of Songkla University, Hat Yai, Songkhla 90110, Thailand.

${ }^{3}$ Department of Electrical Engineering, Faculty of Engineering, Prince of Songkla University, Hat Yai, Songkhla 90112, Thailand.

Received 15 February 2020 • Revised 24 September 2020 • Accepted 24 September 2020 • Published online 25 March 2021

\begin{abstract}
:
Objective: To investigate the cause of a broken metallic tracheostomy tube.
\end{abstract}

Material and Methods: In this study, we performed a fractographic investigation of a broken tracheostomy tube from an elderly patient using surface visualization, scanning electron microscopy, energy dispersive X-ray spectroscopy, and chemical composition analysis using X-ray fluorescent and metallographic testing.

Results: Surface visualization revealed multiple corrosive pits which were confirmed by liquid penetrant testing. Scanning electron microscopy and energy dispersive X-ray spectroscopy revealed the chemical composition of the tube to be an austenitic chromium-nickel-manganese stainless steel alloy. Metallographic analysis suggested that the fracture site originated from the inner surface from intergranular corrosion.

Conclusion: The evidence suggests that the corrosion resistance properties of this material might not be suitable for long term use in the human trachea. Higher grade stainless steel or more frequent device change is recommended.

Keywords: corrosion, stainless steel, tracheostomy tube fracture

Contact: Monthira Tanthanuch, M.D.

Department of Surgery, Faculty of Medicine, Prince of Songkla University,

Hat Yai, Songkhla 90110, Thailand.

E-mail: tmonthir@hotmail.com

(c) 2021 JHSMR. Hosting by Prince of Songkla University. All rights reserved.

This is an open access article under the CC BY-NC-ND license

(http://www.jhsmr.org/index.php/jhsmr/about/editorialPolicies\#openAccessPolicy).
J Health Sci Med Res 2021;39(4):321-329 doi: 10.31584 /jhsmr.2021789 www.jhsmr.org 


\section{Introduction}

A tracheostomy tube is a medical device inserted into the trachea with the primary purpose of establishing and maintaining a patient's airway to ensure the adequate exchange of oxygen and carbon dioxide. A standard tracheostomy tube is usually made of a metal such as stainless steel or silver or a synthetic material such as polyvinylchloride, polyurethane, plastic or silicone. A stainless steel tracheostomy tube is the most popular due to its durability, resistance to bacterial growth including biofilm formation, low level of reactions with human tissue and ease of cleaning as it can be sterilized with heat. However, the properties of stainless steel depend upon the chemical composition of the particular alloy and the manufacturing process.

The stainless steel tracheostomy tube is comprised of three parts, an outer cannula with flange (neck plate), an inner cannula and an obturator. The outer cannula is the outer tube that holds the tracheostomy open. The inner cannula fits inside the outer cannula and can be removed for cleansing by boiling water or hydrogen peroxide. The obturator is used to insert a tracheostomy tube.

The first case of an in-situ fracture of a tracheostomy tube was reported in 1960. ${ }^{1}$ Since then, various fractures have occurred, and various causes of tracheostomy erosion have been suggested, including alkaline tracheal secretions, repeated sterilizations, prolonged and repeated usage and manufacturing factors. ${ }^{2}$ Jacobs and colleagues studied the $\mathrm{pH}$ of respiratory secretions in various disease statuses in humans and reported varying $\mathrm{pHs}$ from 4.2 in the main bronchus of patients with lung cancer to 9.2 in patients with a bronchogenic cyst. ${ }^{3}$ From 1913 to 2017, 82 cases of fractured tracheostomy were reported worldwide, with the majority from India.

The reported fractures have occurred in both metallic and synthetic material tubes and the junction between the cannula body and its flank was the most common fracture site, followed by the shaft of the tube. One study suggested that metallic tracheostomy tubes are more prone to fracturing than synthetic tubes. ${ }^{2}$

In Thailand during the years 2001-2016, there were only three reports of a fractured tracheostomy tube ${ }^{4-6}$, all involving metallic tubes with the fracture site at the shaft. In such cases, a complete fracture can lead to downward migration of a tube fragment to the bronchus, which can cause airway obstruction manifested by cough, choking and even death. Prompt management in such event begins with a bronchoscopy. An open thoracotomy can be necessary in cases in which the foreign body is unreachable by endoscopic means.

Here we report comprehensive physical studies of a tracheostomy tube from a 90-year-old female patient whose tube fractured. The case was a bed-ridden cerebrovascular accident patient who developed persistent choking and cyanosis. A chest $\mathrm{X}$-ray revealed a broken tracheostomy tube with its outer cannula slipped into the left main bronchus. The foreign body was successfully removed by a balloon catheter withdrawal technique. The tube had been in use for 1 year. The aim of this study was to evaluate the physicochemical properties of a broken tracheostomy tube to help lead to a better understanding of the fracture mechanism in such cases.

\section{Material and Methods}

After the fractured tube was successfully removed (Figure $1 \mathrm{~A}$ ), the retrieved cannula was rinsed with tap water and double-packed in Ziploc plastic bags. It was sent to our investigation unit and investigated by (1) liquid penetrant testing using both fluorescent and visible dyes, (2) scanning electron microscopy (SEM), (3) chemical composition analysis using energy-dispersive $\mathrm{X}$-ray spectroscopy (EDX), (4) chemical composition analysis using $X$-ray fluorescence, and (5) metallographic assay. 

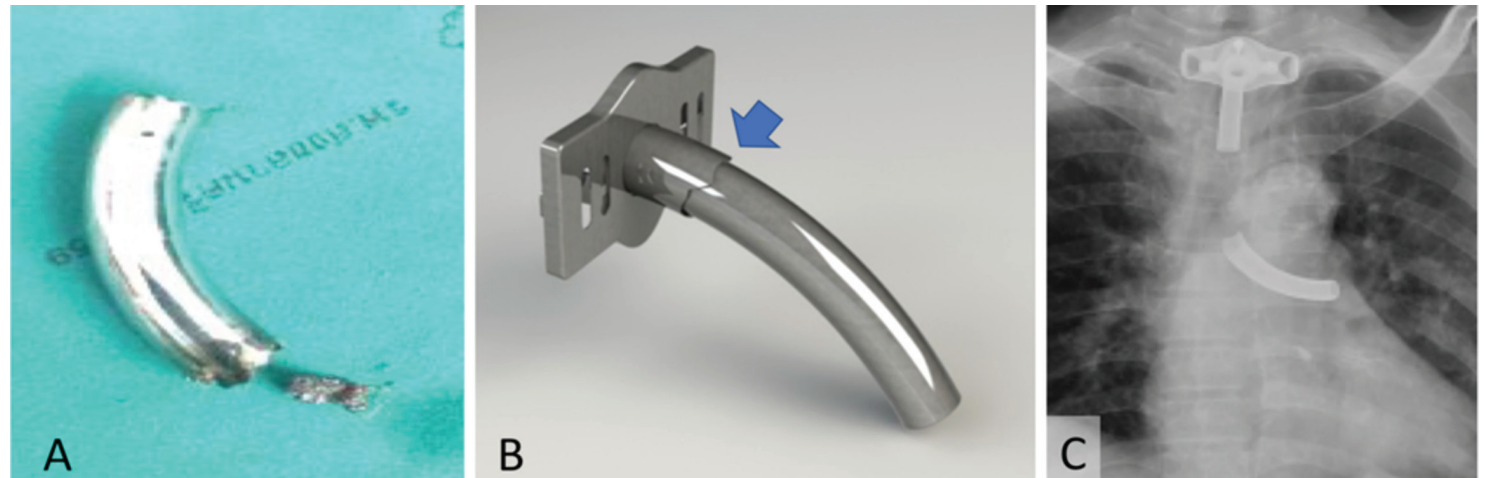

Figure 1 (A) Macroscopic picture of the distal part of the outer tube retrieved from the bronchus; (B) picture of a new fractured tracheostomy indicating the fracture site (arrow); (C) chest x-ray showing the site of the dislodged outer tube in the left main bronchus.

\section{Results}

\section{Visual inspection of the fractured part}

The fractured part of the tracheostomy tube was about $11.5 \mathrm{~mm}$ outer diameter (OD), $0.6 \mathrm{~mm}$ thick and $55 \mathrm{~mm}$ long. It was curved with a polished surface. After cleaning by immersion in absolute alcohol and soft brushing, the surface was seen to be stained with hard brown deposits. Two cracks could be seen, one $22 \mathrm{~mm}$ in length longitudinally with branching tips on the outer curve and the second $12 \mathrm{~mm}$ in length parallel to the larger one (Figure 2A). There was a corroded pit of about $4 \times 2.5 \mathrm{~mm}$ in area and $0.5 \mathrm{~mm}$ in depth near the inner curvature (Figure $2 \mathrm{~B}$ ), with a hole right through the tube of $1 \mathrm{~mm}$ in diameter (Figure $2 \mathrm{C}$ ), apparently originating from the internal surface as the external surface was otherwise uncorroded in this area. The fracture was a brittle type as there were no gross deformities or uneven edges. There was thick brown staining on the inner surface of the device (Figure 2D).

\section{Liquid penetrant test}

By the application of both a fluorescent liquid penetrant and visible dye penetrant, discrete longitudinal linear indications of cracking were found clustered close to the fracture site (Figure 3).

\section{Scanning electron microscope}

Investigation by SEM showed intergranular corrosion along the fracture site. Cracks could be seen along the grain boundary (Figure 4).

\section{Chemical composition analysis}

Chemical composition analysis of deposits from the inner surface using EDX Spectroscopy method indicated that the deposits were a ferrous-rich oxide. There were also significant amounts of carbon, manganese, phosphorus and calcium (Figure 5).

Chemical composition analysis of the retrieved fragment was performed using $X$-Ray fluorescence which indicated that the main composition was austenitic chromium-nickel-manganese stainless steel, specifically $0.15 \%$ carbon $(\mathrm{C}), 1.0 \%$ silicon $(\mathrm{S}), 5.5-7.5 \%$ manganese (Mn), 0.06\% phosphorus (P), 0.03\% sulphur (S), 16.0-18.0\% chromium (Cr) and 3.5-5.5\% nickel (Ni).

\section{Metallographic test}

A micrograph of the retrieved fragment showed an austenitic structure, the typical microstructure of stainless 
steel, with a significant carbide and/or second phase precipitated intergranular structure in the tube. The cracks appeared to originate at the internal surface in an inter- granular pattern. Intergranular corrosion was observed in several tested areas with selective grain loss.

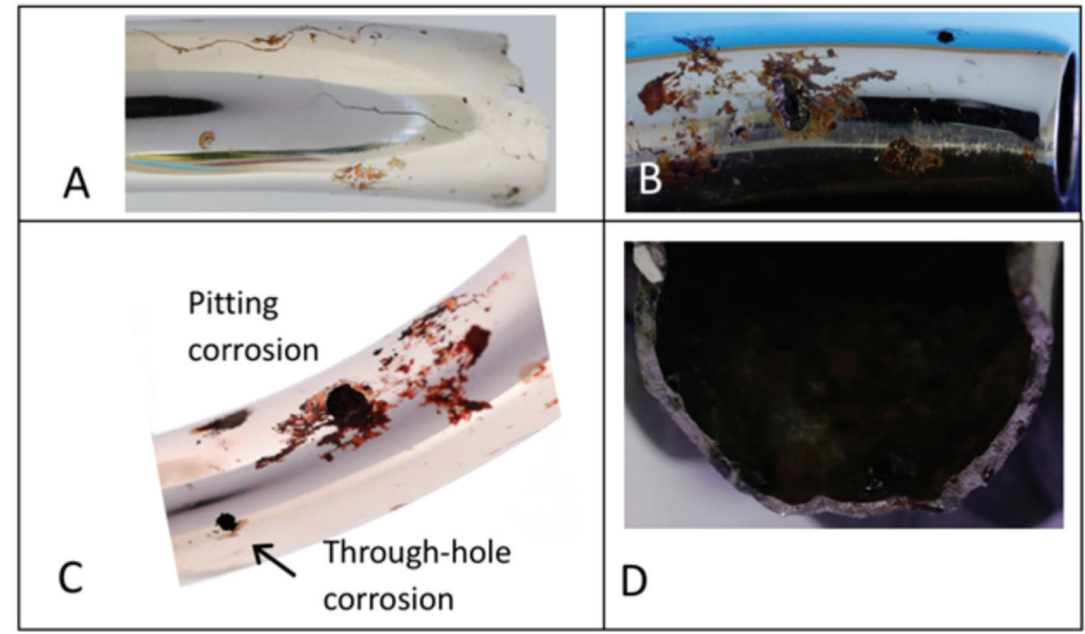

Figure 2 (A) Multiple longitudinal cracks and deposits on the external surface. (B) Pitting corrosion with deposits on the external surface. (C) A full-thickness corrosion hole on the outer curvature. (D) Brittle fracture with no deformation of the tube
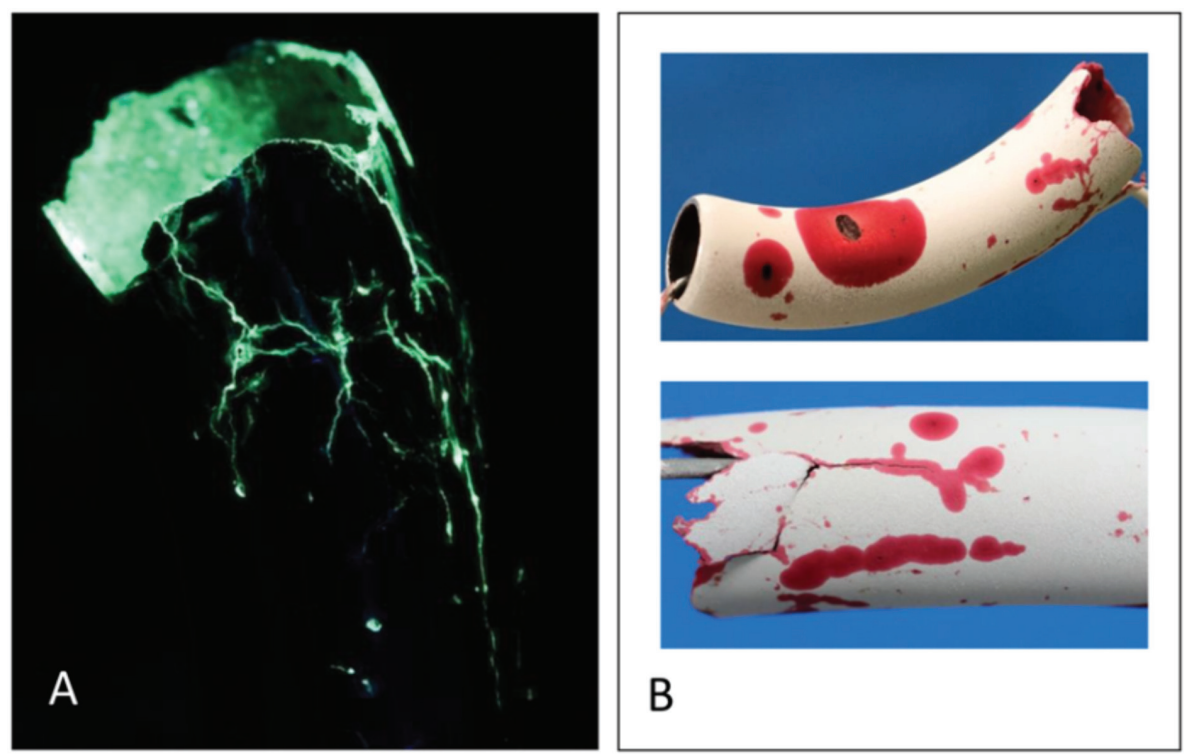

Figure 3 Picture from the liquid penetrant test and fluorescent dye penetrant test at the external surface. (A) With the fluorescent dye penetrant at the external surface, several longitudinal linear cracks were detected around the fracture. (B) With the liquid penetrant test; visible dye penetrant, at the external surface, several linear and round markings were detected. 

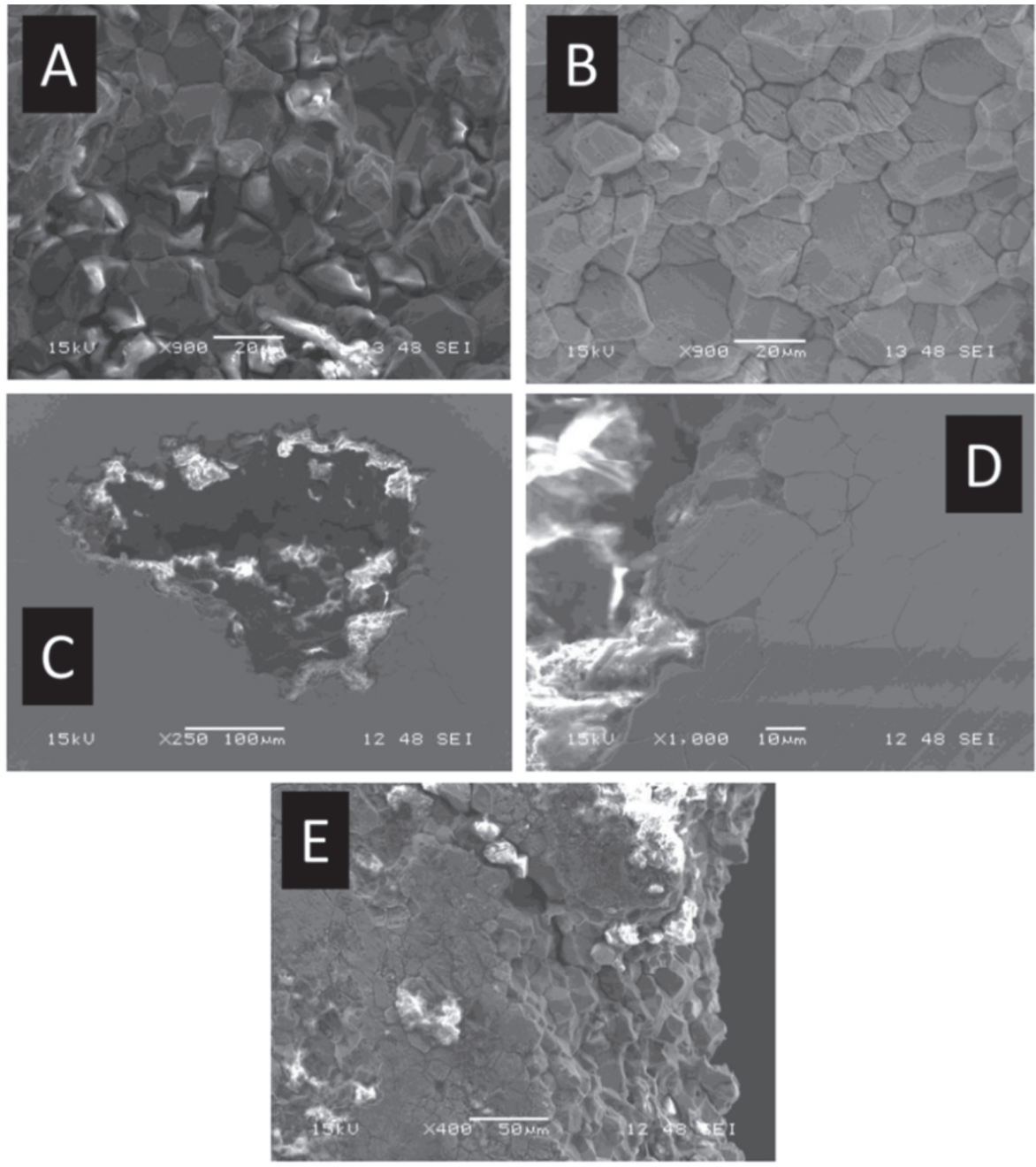

Figure 4 (A-B) Scanning electron microscopy (SEM) images showing the intergranular corrosion effects at the fracture surface. (C-D) SEM images of the external surface near the pit with intergranular attack. (E) The internal surface close to the fracture also shows inter-granular corrosion effects. 
Spectrum processing:

Peak possibly omitted $: 2.351 \mathrm{keV}$

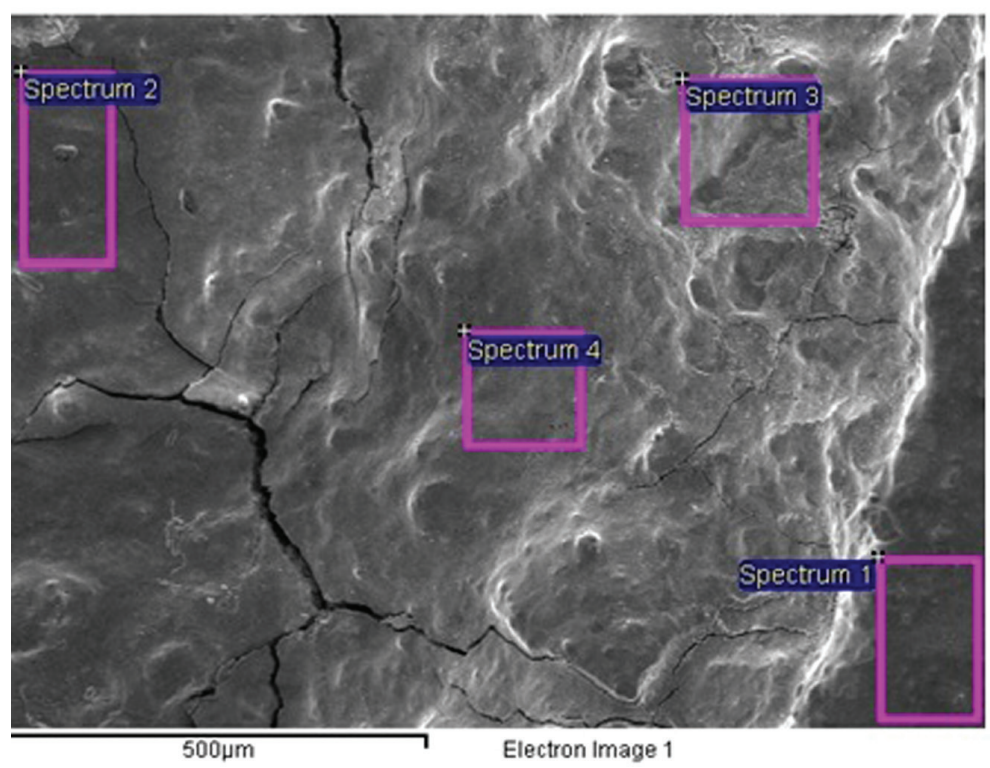

Processing option: All elements analyzed

(Normalised)

Number of iterations $=3$

Standard:

C CaCO3 1-Jun-1999 12:00 AM

O $\mathrm{SiO} 2$ 1-Jun-1999 12:00 AM

$\mathrm{Cr} \mathrm{Cr}$ 1-Jun-1999 12:00 AM

Mn Mn 1-Jun-1999 12:00 AM

$\mathrm{Fe}$ Fe 1-Jun-1999 12:00 AM

\begin{tabular}{l|ll}
\hline Element & Weight $\%$ & Atomic\% \\
& & \\
$\mathrm{C} \mathrm{K}$ & 12.41 & 23.63 \\
$\mathrm{O} \mathrm{K}$ & 39.39 & 56.30 \\
$\mathrm{Cr} \mathrm{K}$ & 10.10 & 4.44 \\
$\mathrm{Mn} \mathrm{K}$ & 3.14 & 1.31 \\
$\mathrm{Fe} \mathrm{K}$ & 34.96 & 14.32 \\
& & \\
Totals & 100.00 & \\
\hline
\end{tabular}

$\mathrm{KeV}=$ kiloelectron volt, $\mathrm{C}=$ carbon, $\mathrm{O}=$ =xygen, $\mathrm{Cr}=$ chromium, $\mathrm{Mn}=$ manganese, $\mathrm{Fe}=$ ferrum (Iron), $\mathrm{CaCO}=$ calcium carbonate, $\mathrm{SiO} 2=$ silicon dioxide, K=kalium (Potassium)

Figure 5 Energy dispersive X-ray spectroscopy report of deposits from the inner surface 

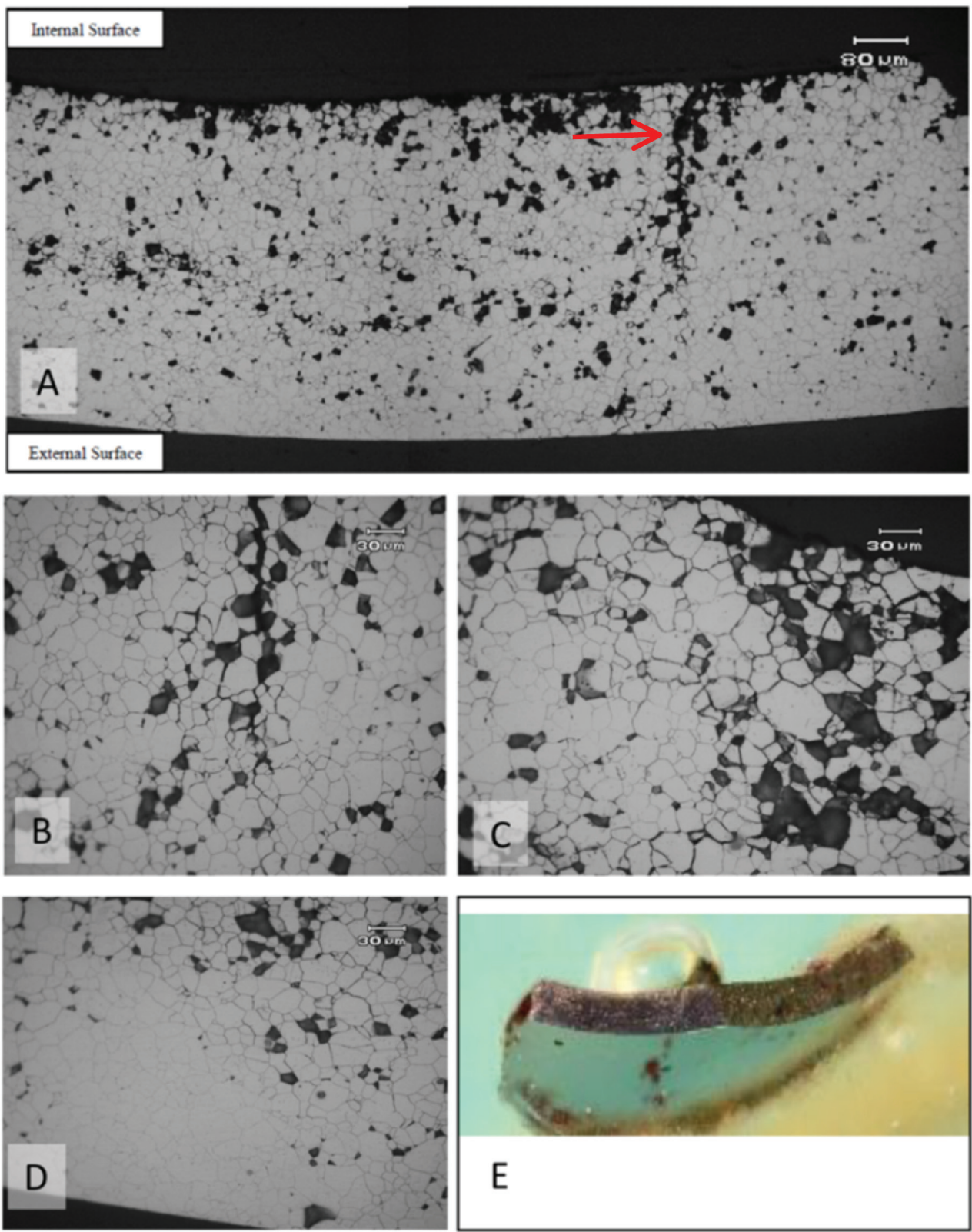

Figure 6 Micrographic pictures of the tube: (A) crack propagation along the internal surface (arrow), (B) intergranular corrosion at the external surface and, (C) the internal surface, (D) carbide and/or second phase precipitation at the grain boundary, $(E)$ the fracture surface of the retrieved fragment. 


\section{Discussion}

Stainless steel is a common material used in various surgical devices. Medical grade stainless steel usually requires a high percentage of chromium and specific surface treatment to ensure a stable oxide layer and prevent corrosion. ${ }^{7,8}$ In this study we performed a comprehensive physicochemical study of a broken tracheostomy tube. The macroscopic appearance suggested that the device slowly deteriorated over a relatively long period. A combination SEM-EDX study found chemical traces of steel oxide, which suggest that intergranular corrosion caused by oxidation occurred in the stainless steel used in the manufacture of this particular tracheostomy device.

Chemical composition analysis with EDX revealed that the device had higher manganese $(\mathrm{Mn})$ and lower nickel $(\mathrm{Ni})$ content than conventional stainless steel with a $\mathrm{Cr}-\mathrm{Ni}$ alloy base. One interpretation of this finding is that the manufacturer of this device attempted to reduce their production costs by replacing nickel with manganese for the austenite creation, which had the downside of reducing corrosion resistance. Corrosion resistance of surgical grade stainless steel is normally enhanced by alloying it with molybdenum (Mo) (stainless steel 316).

Most fractographic studies of stainless steel devices used in a human body which have failed have reported that the failure was usually a result of fatigue at a sharp corner. ${ }^{9}$ One study on $\mathrm{Cr}$-Mo alloy with a low carbon stainless steel content (stainless steel 316L) supracondylar plate reported that the material failed only when excess tensile stress was applied. ${ }^{10}$ In our case, the evidence suggests that corrosion was the major cause of the fracture rather than stress cracking. From this evidence, we suggest that $\mathrm{Cr}-\mathrm{Mn}$ alloy stainless steel might not be suitable for long term intracorporeal use.

\section{Conclusion}

Our study analyzed the physicochemical properties of a fractured tracheostomy device and found that the failure was likely the result of corrosive damage that occurred over a long period of time. The chemical profile analysis found that the device was based on a $\mathrm{Cr}-\mathrm{Mn}$ alloy rather than the recommended $\mathrm{Cr}-\mathrm{Ni}$ alloy (food grade stainless steel) or Cr-Mo alloy (surgical grade stainless steel), thus the appropriateness of the material used in the manufacturing of this device is questionable. In cases of an intracorporeal device intended for long term use, such as a tracheostomy tube, one expert has suggested careful inspection of the device by the health care provider during each maintenance visit and replacement every 6 months. ${ }^{6}$

\section{Acknowledgement}

The authors thank Chevlon Thailand Exploration and Production, Ltd., for their support through allowing the use of their laboratory for failure analysis of the tracheostomy tube.

\section{Conflicts of interest}

The author declare no conflicts of interest.

\section{References}

1. Bassoe $\mathrm{HH}$, Boe J. Broken tracheotomy tube as a foreign body. Lancet 1960;1:1006-7.

2. Herrag M, Sajiai H, Rochdi Y, Lahmiti S, Dannaoui Y, Raji A, et al. Flexible bronchoscopic removal of a fractured metallic tracheostomy tube. J Bronchology Interv Pulmonol 2011;18: 164-7.

3. Jacobs SE. Chemical disinfection. Some aspects of the dynamics of disinfection. J Pharm Pharmacol 1960;12(Suppl): $9-18$.

4. Srirompotong S, Kraitrakul S. Fractured inner tracheostomy tube: an unusual tracheobronchial foreign body. Srinagarind Med J 2001;16:223-5. 
5. Piromchai $P$, Lertchanaruengrit $P$, Vatanasapt $P$, Ratanaanekchai

$\mathrm{T}$, Thanaviratananich S. Fractured metallic tracheostomy tube in a child: a case report and review of the literature. J Med Case Rep 2010;4:234.

6. So-Ngern A, Boonsarngsuk V. Fractured metallic tracheostomy tube: A rare complication of tracheostomy. Respir Med Case Rep 2016;19:46-8.

7. Hryniewicz T, Rokicki R, Rokosz K. Corrosion characteristics of medical-grade AISI Type 316 L stainless steel surface after electropolishing in a magnetic field. Corrosion 2008;64:660-5.
8. Ulrich Heubner W. Stainless steel - when health comes first. Brussels: Euro Inox; 2009

9. Guerra-Fuentes L, García-Sánchez E, Juárez-Hernández A, Hernández-Rodríguez M. Failure analysis in 316L stainless steel supracondylar blade plate. Eng Fail Anal 2015;57: 243-7.

10. Hernandez-Rodriguez A. Failure analysis in $316 \mathrm{~L}$ stainless steel supracondylar blade plate. Eng Fail Anal 2015;57:243-7. 